\title{
Study of Long-lived Heavy Charged Particles Produced in PP Collisions at Energy $13 \mathrm{TeV}$
}

\author{
Nguyen Mau Chung* \\ Faculty of Physics, VNU University of Science, 334 Nguyen Trai, Hanoi, Vietnam \\ Received 08 July 2017 \\ Revised 30 August 2017; Accepted 15 September 2017
}

\begin{abstract}
We are interested in long-lived heavy charged particles because they would be possible SUSY particle candidats. This paper shows our preliminary results of long-lived heavy charged particles generation using PYTHIA 8. More than $10^{7}$ events have been generated with $p p$ collisions at energy in the center of mass $\sqrt{s}=13 \mathrm{TeV}$ and about $3.27 \times 10^{5}$ long-lived heavy charged particles candidates have been found in the geometric acceptance of the LHCb detector. Long-lived heavy charged particles has mass $1.025 \mathrm{TeV} / \mathrm{c}^{2}$ and lifetime $\tau=157.7$ nanoseconds, therefore they can travel throughout all subdetectors. We try not only calculate their acceptance in function of transverse momentum and rapidity but also combine a pair of candidats with opposite charge in order to reconstruct their invariant mass. In the next step, we have intention to identify stau using the informations from subdetectors such as the inner tracker and the muon chambers.
\end{abstract}

Keywords: Long-lived massive stau, superpatner, p-p collisions.

\section{Introduction}

In this paper, we present our new research orientation concerning long-lived heavy charged particles that would be produced in hadron collisions at very high energy. Since several years, Vietnamese physicists of the "Groupe de Physique des Hautes Energies" (GPHE) have participated in the LHCb experiment with the help of the Ecole Polytechnique Federale de Lausanne (EPFL). Our group chooses the decays of B meson as one of study subjects because the neutral B meson systems play a predominant role in investigating $\mathrm{CP}$ violation and any deviation from the Standard Model (SM). We have also studies strangeness production because this process can reveal the particle production mechanism in the particle collision. Long-lived heavy charged particles will be a new and interesting study subject especially for the younger members in our groupe who have a chance to study some physics phenomena beyond the Standard Model.

\footnotetext{
* Tel.: 84- 983381957.

Email: chungnm@vnu.edu.vn

https//doi.org/ 10.25073/2588-1124/vnumap.4219
} 


\section{Physics motivation}

There are several reasons to expect Beyond Standard Model (BSM) physics to emerge at the TeV scale being explored at the Large Hadron Collider (LHC). The first general argument is simply that there are many free parameters in Standard Model and a more unified theory should contrain fewer. An important argument that points to new physics being manifested at the $\mathrm{TeV}$ scale is known as the hierarchy problem. The most popular solution to the hierarchy problem is to invoke SuperSymmetry (SUSY). This is a symmetry that transform a boson into fermion and vice versa. This implies that every SM particle must have a superpatner with spin differing by $\frac{1}{2}$. Although there is currently no direct evidence for SuperSymmetry, there are some suggetive hints that it might be a valid low-energy theory.

Several extensions of the Standard Model propose the existence of charged long-lived massive particles that can travel through a detector without decaying. These particles can have long lifetimes for a variety of reasons, e.g. a new (approximately) conserved quantum number, a weak coupling or a limited phase. Following some of these above theories, the next-to-lightest supersymmetric particle can be a long-lived stau with a mass of the order of $100 \mathrm{GeV} / \mathrm{c}^{2}$ or higher. Several experiments have search up to masse about $700 \mathrm{GeV} / \mathrm{c}^{2}$, but no evidence is found for the production of such long-lived state. We begin this research expecting these states exist at the mass scale aroud 1 or $2 \mathrm{TeV}$.

\section{Analysis strategy}

For charged long-lived massive particle, strong interaction are neglected in comparison with electromagnetics one, therefore they lose their energy mainly via ionisation. With a kinetic energy above about $5 \mathrm{GeV}$, in a detector such as LHCb [1,2], these particles should be able to traverse the muon chambers. Because they would often be produced with a relatively low velocity, we could identify them by their time-of-flight, and by their specific energy loss, $d E / d x$, in the different detectors. Especially, Cherenkov radiation would be absent in Cherenkov counters in the case these detectors are tuned for ultra-relativistic particles.
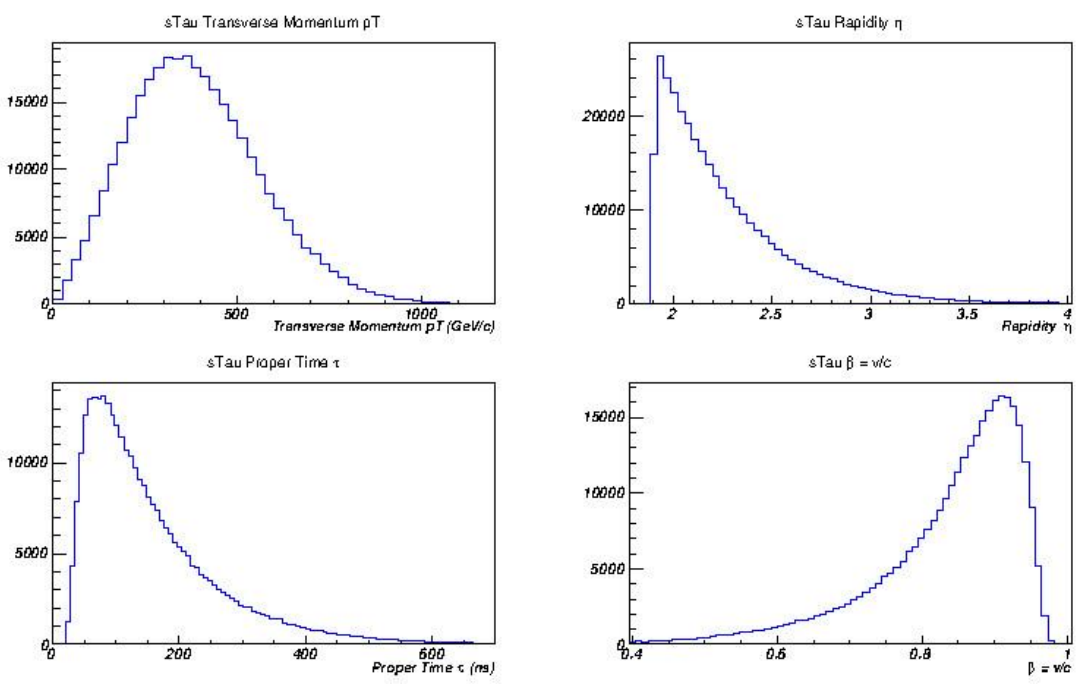

Figure 1. Typical distributions of generated stau from pp collisions at energy $13 \mathrm{TeV}$. 
We have intention to perform a search for heavy long-lived charged particles using proton-proton collisions collected at $\sqrt{s}=13 \mathrm{TeV}$ with the LHCb detector because we have a larger prodution crosssection at this energy scale. The search is mainly based on the response of the ring imaging Cherenkov detectors that allow us to distinguish the heavy, slow-moving particles from muons. The results are expressed as the Drell-Yan production of pairs of long-lived particles, with both particles in the LHCb pseudorapidity acceptance, $1.8<\eta<4.9$.

As a first step, Monte Carlo events have been generated with $p p$ collisions at energy in the center of mass $\sqrt{s}=13.0 \mathrm{TeV}$. Long-lived heavy charged particles has mass in order of $\mathrm{TeV} / \mathrm{c}^{2}$ and lifetime about several hundred nanosecond have been produced in these collisions. Analysis these Monte Carlo data allow us to find the optimum cut criteria that would be used to analyse the real data being collected at LHCb detectors.

\section{Prelimilary results}

Using the minimal gauge-mediated supersymmetry breaking (mGMSB) model, we suppose that stau pair are producted via a Drell-Yan process in proton proton collision at high energy. These pairs originating from cascade decays of heavier particles that are explicitly not considered and are expected to be found in the near future. The mGMSB model has six parameters [3]: the SUSY breaking scale $(\Lambda)$, the mass scale of the SUSY loop messengers $M_{m}$, the number of messenger supermultiplets $\left(\mathrm{N}_{5}\right)$, the ratio of the vacuum expectation values of the two neutral Higgs fields $(\tan \beta)$, the sign of the Higgs mass parameter $(\mu)$, and the parameter $\mathrm{C}_{\text {grav }}$, which affects the gravitino mass.

We used the Spheno spectrum generator to compute the masses of the stau as a function of the above six parameters. We try to vary the value of $\Lambda$ in order to determines the stau mass and lifetime, which is of the order of 100 nanoseconds. In our simulation, the following parameters $\mathrm{N}_{5}=3, \tan \beta=$ $35, \mu>0, M_{m}=2 \Lambda$, and $C_{\text {grav }}=8000$ are fixed. In this study, the stau is considered stable, because they can travell throughout our detector.
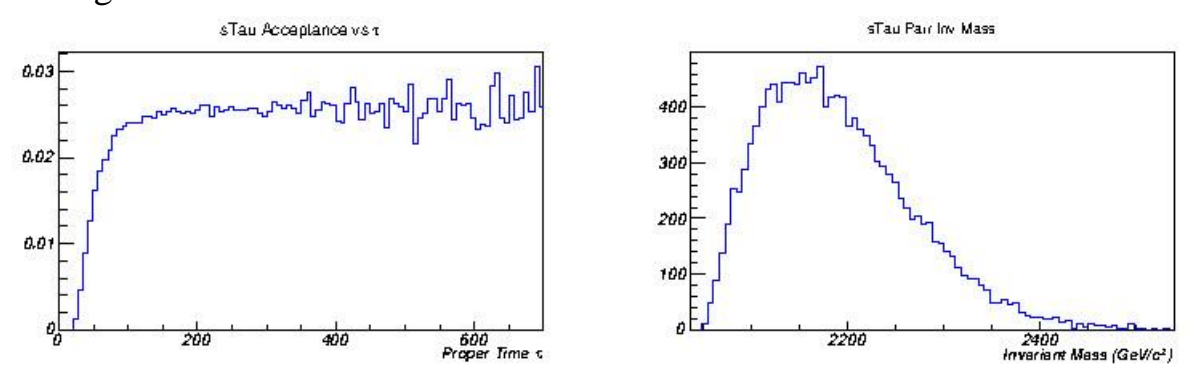

Figure 2. Stau acceptance as a function of the proper time and the invariant mass of stau pair.

We are interested in large stau masses because the production of these particle increase with theses masses, on the other hand our detector's acceptance is reduced. For larger stau masses, the Drell-Yan process results in a lower forward boost of the stau pair, subsequently the pair opening angle in the detector frame increase. In this case, particles have a higher probability to escape the LHCb geometrical acceptance, therefore the acceptance will decrease.

Fully simulated signal samples, with masses varying from 1000 to $1100 \mathrm{GeV} / \mathrm{c}^{2}$, have been produced for $p p$ collisions at $\sqrt{s}=13 \mathrm{TeV}$. The stau pairs are generated by Pythia[4], only stau particles in the fiducial range $1.8<\eta<4.9$ are passed to detector simulation in the next step. Some 
typical distributions generated stau in the fiducial range have been shown in the figure 1 . Physics parameters (energy, rapidity, transverse momentum and proper time) of several candidats are displayed on the table 1 .

The figure 2 shows the stau acceptance (the fraction of stau within the fiducial range) as a function of the proper time $\tau$. We also try to combine a pair of candidats with opposite charge in order to reconstruct their invariant mass expecting to find the heavier particles whose cascade decay products are stau.

Table 1. Candidates of stau and antistau

\begin{tabular}{lcclll}
\hline Event & Particle & $\mathrm{E}(\mathrm{GeV})$ & $\mathrm{P}_{\mathrm{t}}(\mathrm{GeV})$ & Rapidity & $\tau(\mathrm{ns})$ \\
\hline 642 & stau & 1161.96 & 251.56 & 2.332 & 109.33 \\
803 & stau & 2332.63 & 580.64 & 1.957 & 285.55 \\
1012 & stau & 1795.84 & 412.52 & 1.947 & 225.04 \\
1255 & stau & 2120.69 & 498.98 & 1.988 & 90.53 \\
1449 & stau & 2141.81 & 547.39 & 1.905 & 104.13 \\
717 & antistau & 2675.72 & 613.85 & 2.070 & 328.49 \\
854 & antistau & 1349.06 & 206.00 & 2.272 & 144.16 \\
1206 & antistau & 2115.07 & 524.60 & 1.933 & 106.82 \\
1449 & antistau & 2429.54 & 509.75 & 2.143 & 387.84 \\
1617 & antistau & 1322.76 & 120.22 & 2.627 & 114.43 \\
\hline
\end{tabular}

\section{Conlussion}

The GPHE has worked successfully in choosing the new and interesting study subject concerning long-lived heavy charged particles produced in hadron collision at the TeV scale. Ten million longlived heavy charged particles which has mass $1.025 \mathrm{TeV} / \mathrm{c}^{2}$ and lifetime $\tau=157.7$ nanoseconds have been generated and about $3 \%$ of them have been found in the geometric acceptance of the $\mathrm{LHCb}$ detector. No peak above the background expectation is observed in the invariant mass reconctructed from a pair of candidats with opposite charge. After finding the optimum cut criteria, our group will perform the next important step : analysing the real data being collected at LHCb detectors.

\section{Acknowledgements}

The author would like to thank the Faculty of Basic Sciences of the Swiss Federal Institute of Technology in Lausanne for having greeted him as a academic host in its Laboratory for High Energy Physics during the summer of 2016.

\section{References}

[1] LHCb Collaboration, A.A. Alves Jr. et al., "The LHCb detector at the LHC". JINST 3, S08005 (2008).

[2] LHCb Collaboration, R. Aaij et al., "LHCb detector performance". Int. J. Mod. Phys. A 30, 1530022 (2015).

[3] S. P. Martin, A supersymmetry primer. arXiv:hep-ph/9709356.

[4] T. Sjostrand, S. Mrenna, P. Skands. JHEP 05, 026 (2006). 